\title{
MRI Texture-Based Recognition of Dystrophy Phase in Golden Retriever Muscular Dystrophy Dogs. Elimination of Features that Evolve along with the Individual's Growth
}

\section{Dorota Duda ${ }^{1}$}

${ }^{1}$ Faculty of Computer Science, Bialystok University of Technology, Poland

\begin{abstract}
The study investigates the possibility of applying texture analysis (TA) for testing Duchenne Muscular Dystrophy (DMD) therapies. The work is based on the Golden Retriever Muscular Dystrophy (GRMD) canine model, in which 3 phases of canine growth and/or dystrophy development are identified: the first phase (0-4 months of age), the second phase (from over 4 to 6 months), and the third phase (from over 6 months to death). Two differentiation problems are posed: (i) the first phase vs. the second phase and (ii) the second phase vs. the third phase. Textural features are derived from T2-weighted Magnetic Resonance Imaging (MRI) images. In total, 37 features provided by 8 different TA methods (statistical, filter-based, and model-based) have been tested. The work focuses on finding such textural features that evolve along with the dog's growth. These features are indicated by means of statistical analyses and eliminated from further investigation, as they may disturb the correct assessment of response to treatment in dystrophy. The relative importance of each remaining feature is then assessed with the use of the Monte Carlo (MC) procedure. Furthermore, feature selection based on the MC procedure is employed to find the optimal subset of age-independent features. Finally, three classifiers are used for evaluating different sets of textural features: Adaptive Boosting (AB), back-propagation Neural Network (NN), and nonlinear Support Vector Machines (SVM). The best subsets of age-independent features ensure $80.0 \%$ and $78.5 \%$ of correctly identified phases of dystrophy progression, for the first (i) and second (ii) differentiation problem respectively.
\end{abstract}

\section{Introduction}

Duchenne muscular dystrophy (DMD) is a hereditary genetic disorder caused by the absence or reduced expression of dystrophin, a protein that plays a key structural role in muscle fiber function (Guiraud et al., 2015). The disease is found predominantly in male children and young men, and results in a progressive destruction of all the striated muscles. The affected 


\section{Dorota Duda}

individuals usually die by the age of 30 , due to respiratory failure (LoMauro et al., 2015) or cardiomyopathy (Spurney, 2011). So far, many extensive attempts have been made to elaborate therapies for DMD (Salmaninejad et al., 2018; Shieh, 2015); however, none of them turned out to be effective enough. For the time being, there is no cure and various healthcare practices can only improve the life expectancy or alleviate the patient's suffering (Birnkrant et al., 2018a, 2018b). In view of the above, the search for an efficient DMD therapy is continually of vital interest.

An important problem encountered while elaborating therapeutic strategies is the right choice of protocols for the assessment of treatment effects. Seeing that such an assessment must often be made repeatedly over a period of time, the use of invasive procedures, even highly effective ones, is undesirable here. For instance, biopsies for histopathological analysis can alter muscle integrity and weaken the muscles, already damaged by the disease. In turn, other measurement tools, e.g. evaluating motor function, muscle strength, respiratory function or disability, are not entirely satisfactory and sometimes difficult to perform on non-ambulant patients (EMA, 2015). In this context, great hope is placed in Magnetic Resonance Imaging (MRI) techniques, which are non-invasive and can provide relevant information about the stage of neuromuscular disorders (Finanger et al., 2012). Nevertheless, as a proper interpretation of image content is not a trivial task, muscle imaging is still used as complementary to clinical and electrophysiological examination in neuromuscular disease (Simon et al., 2016). Its role could be increased by using (semi)automated computer-aided methods for the analysis and interpretation of muscle images. Those based on texture analysis (TA) seem to be particularly promising (de Certaines et al., 2015; Lerski et al., 2015).

Some works assessing the possibility of applying texture analysis for muscular (dystrophic) tissue characterization have already been undertaken, using different animal models, dogs- or mice-based. They focused either on differentiation between groups of healthy and dystrophic muscles at several stages of animal growth and/or disease progression (Duda et al., 2015; Fan et al., 2014; Wang et al., 2013; Yang et al., 2015), or differentiation among several phases of dystrophy development in affected animals (Duda et al., 2016; Martins-Bach et al., 2015). These works showed that the use of textural features as tissue descriptors can lead to a relatively high distinguishability of the considered muscle groups (see, for example, the study by Duda et al. (2015)) and that textural features can demonstrate a better potential, in comparison with other, non-texture-based, MRI biomarkers (Fan et al., 2014; Martins-Bach et al., 2015). Furthermore, they allowed to 
determine which texture analysis methods can provide the most useful information, in terms of the considered discrimination process. In general, highly effective features turned out to be those derived from the co-occurrence matrices (Haralick et al., 1973), the run-length matrices (Galloway, 1975), the gray level difference matrices (Weszka et al., 1976), or the gray level histogram.

It should be noted that evolution of muscle texture properties over time could result from (at least) two processes taking place simultaneously: the individual's growth and disease progression. However, the afore-cited works did not evaluate how each of these two processes influences the characteristics of muscle texture separately. If a textural feature varied significantly form one dystrophy phase to another, or if its values were significantly different for healthy and affected animals, the feature was considered as "potentially useful" in the differentiation between the examined muscle classes. It was not estimated whether or how the feature value depended on the animal's age itself, while differentiating among several phases of dystrophy development (progressing with age) in affected animals. If texture analysis was to be used to test the therapy's effects, special attention should be paid to features whose value evolves along with the individual's growth. Ignoring such an evolution could alter the correct assessment of dystrophy progression or its response to treatment. On the other hand, constructing a tool for therapy assessment based on age-dependent features could be difficult if it is not known how slowing down the disease can influence the summary changes in feature values.

The first objective of the present study is to evaluate which textural features may significantly change with the individual's growth. Such features are indicated by means of statistical analyses and eliminated from further investigation. The second objective is to measure the relative importance of each remaining (age-independent) feature in identifying the dystrophy phase. This task is accomplished by applying the modified Monte Carlo (MC) procedure. Furthermore, the study assesses the best possible differentiation of dystrophy phases that could be achieved solely with the use of features that do not depend on the individual's age. At this stage, apart from the whole set of age-independent features, its different subsets, found based on the modified Monte Carlo procedure, are also examined. The subsets are tested with the use of three classifiers: the Adaptive Boosting - AdaBoostM1 (AB), the back-propagation Neural Network (NN), and nonlinear Support Vector Machines (SVM).

The study is based on the Golden Retriever Muscular Dystrophy (GRMD) canine model, which is acknowledged to be the closest to hu- 


\section{Dorota Duda}

man DMD and commonly used in research on dystrophy progression or therapy trials (Kornegay, 2017). In the model, three phases of canine growth and/or dystrophy development are distinguished, in reference to histological changes in muscle structure, caused by disease progression (de Certaines et al., 2015): the first phase (0-4 months of age), the second phase (from over 4 to 6 months), and the third phase (from over 6 months to death). Due to the fact that it is still relatively difficult to differentiate among the three considered dystrophy phases (Duda et al., 2016), this preliminary work focuses on two-class classification problems, distinguishing only between adjacent phases: (i) the first phase vs. the second phase and (ii) the second phase vs. the third one. Textural features are derived from T2-weighted MRI images of canine hindlimb muscles. In total, 37 features obtained with eight different TA methods (statistical, filter-based, and model-based) are tested.

\section{Materials and Methods}

Four experiments were planned and carried out separately for each differentiation problem, i.e. for (i) the first phase vs. the second phase and (ii) the second phase vs. the third phase. The objective of the first (preliminary) experiment was to determine whether it was at all possible to distinguish among healthy dogs at different phases of their growth. At this step, all the possible textural features (37 in total) were used. In the second experiment, only healthy dogs were considered and statistical analyses were performed in order to find the features whose values could evolve along with the dog's growth. Such features were excluded from further analyses. In the third experiment, one concerning dystrophic dogs, the relative importance (in terms of the discrimination process) of each remaining feature was evaluated and feature selection was performed to find optimal subsets of age-independent features. Finally, in the fourth experiment, different classifiers were used to examine which subsets of age-independent features can ensure the best possible identification of the dystrophy phase in GRMD dogs. The results obtained at this step were compared to those corresponding to the use of all the initially tested features (age-dependent and age-independent put together).

Creation of the Data Base. The experiments were conducted on a total of 422 T2-weighted MRI images, derived from five GRMD dogs and five healthy controls. The dogs were bred in a dedicated gene therapy 
facility at the National Veterinary School of Alfort, France and were imaged at the Nuclear Magnetic Resonance Laboratory of the Institute of Myology in Paris, France. All the procedures applied to the dogs during the course of the trial were carried out in conformity with the Guide for the Care and Use of Laboratory Animals (National Research Council, 2011) and approved by the Institutional Animal Use and Care Committee, respecting the European legislation on laboratory animals and animal studies.

All the details concerning the acquisition protocols were fully described by Thibaud et al. (2012). According to their specification, images were acquired on a 3T Siemens Magnetom Trio TIM imager/spectrometer (Siemens Healthcare, Erlangen, Germany) with a standard, circularly polarized extremity coil. The in-plane resolution was $0.56 \mathrm{~mm} \times 0.56 \mathrm{~mm}$, the slice thickness was $3 \mathrm{~mm}$, and the inter-slice gap was $7.5 \mathrm{~mm}$. The slice orientation was axial with respect to the long axis of the muscle. As for the considered series of T2-weighted images, the repetition time $(T R)$ was 3,000 ms, the first echo time (TE1) and the second echo time (TE2) were, respectively, $6.3 \mathrm{~ms}$ and $50 \mathrm{~ms}$. The size of all images was $240 \times 320$ pixels.

For each dog, between 3 and 5 examinations were performed over a maximum of 14 months, which amounted to 38 examinations in total. For the needs of the present study, each examination was assigned to one of the three considered phases of canine growth and/or dystrophy development. This attribution was based on the age of the dog at the time of examination. It was assumed that both the dog's development and the course of the disease were fairly similar across different dogs. In total, 14, 9, and 15 examinations were assigned to the first, second and third phase, respectively. The exact examination moments, with respect to the dog's age, are marked for each dog in Table 1.

Only one series of T2-weighted images per examination was selected for the study. Each series contained between 12 and 14 images. A small number of images located at the very beginning or end of the series were not considered because it was impossible to identify any quite large and well visible muscle areas on them. On the remaining images, up to four different types of muscles were segmented: the Extensor Digitorum Longus (EDL), the Gastrocnemius Lateralis (GasLat), the Gastrocnemius Medialis (GasMed), and the Tibial Cranialis (TC). If a given muscle was visualized in the image, two Regions of Interest (ROIs) were designated for it, one for each limb - left and right. Only ROIs with the size of at least 100 pixels were considered suitable for analysis. 


\section{Dorota Duda}

Table 1. The examination moments (marked with ' $E$ '), with respect to the dog's age given in months. Each examination is assigned to one of the three phases of canine growth and/or dystrophy development. Each row of the table corresponds to a different dog

\begin{tabular}{|c|c|c|c|c|c|c|c|c|c|c|c|c|c|c|c|}
\hline \multirow{3}{*}{$\begin{array}{c}\text { cohort } \\
\text { type }\end{array}$} & \multirow{3}{*}{$\begin{array}{l}\text { dog's: } \\
\text { no./age }\end{array}$} & \multicolumn{14}{|c|}{ phase of canine growth and/or dystrophy development } \\
\hline & & \multicolumn{4}{|c|}{ first } & \multicolumn{2}{|c|}{ second } & \multicolumn{8}{|c|}{ third } \\
\hline & & 1 & 2 & 3 & 4 & 5 & 6 & 7 & 8 & 9 & 10 & 11 & 12 & 13 & 14 \\
\hline \multirow{5}{*}{ GRMD } & 1 & - & $\mathrm{E}$ & - & $\mathrm{E}$ & - & $\mathrm{E}$ & - & $\mathrm{E}$ & - & - & - & - & - & - \\
\hline & 2 & - & - & - & $\mathrm{E}$ & $\mathrm{E}$ & - & - & $\mathrm{E}$ & - & - & - & - & - & - \\
\hline & 3 & - & $\mathrm{E}$ & - & - & $\mathrm{E}$ & - & - & - & $\mathrm{E}$ & - & $\mathrm{E}$ & - & - & - \\
\hline & 4 & - & - & - & $\mathrm{E}$ & $\mathrm{E}$ & - & - & $\mathrm{E}$ & - & - & $\mathrm{E}$ & - & - & - \\
\hline & 5 & - & $\mathrm{E}$ & - & $\mathrm{E}$ & - & $\mathrm{E}$ & - & - & - & - & - & - & - & - \\
\hline \multirow{5}{*}{ healthy } & 1 & - & - & - & - & $\mathrm{E}$ & - & - & $\mathrm{E}$ & - & - & $\mathrm{E}$ & - & - & - \\
\hline & 2 & - & $\mathrm{E}$ & $\mathrm{E}$ & - & $\mathrm{E}$ & - & - & $\mathrm{E}$ & - & - & - & - & - & $\mathrm{E}$ \\
\hline & 3 & - & $\mathrm{E}$ & - & $\mathrm{E}$ & - & $\mathrm{E}$ & - & $\mathrm{E}$ & - & - & - & $\mathrm{E}$ & - & - \\
\hline & 4 & - & - & - & $\mathrm{E}$ & - & - & - & $\mathrm{E}$ & - & - & $\mathrm{E}$ & - & - & - \\
\hline & 5 & - & $\mathrm{E}$ & $\mathrm{E}$ & - & $\mathrm{E}$ & - & - & $\mathrm{E}$ & - & - & - & - & - & - \\
\hline
\end{tabular}

Figure 1 shows two sample T2-weighted MRI images, used in the experiments. The images were acquired on one GRMD dog (the left one) and one healthy control (the right one), at the age of 8 months. Delineated ROIs cover the four types of muscles considered in the study: EDL, GasLat, GasMed, and TC. Tables 2 and 3 contain, respectively, the numbers of corresponding ROIs and the average ROIs' sizes given separately for each cohort type (GRMD, healthy), each muscle, and each phase of canine growth and/or disease development.

As the average pixel value of the reference object (placed in the image view) varied significantly between different images, even those belonging to the same series, images had to be pre-processed. The pre-processing consisted in linear transformation of image pixel values, so that the reference object was of the same average pixel value on all the resulting images. After completing the procedure, the images were converted from the initial Analyze format to the 8-bit BMP format. In the course of the conversion, only a part of the full range of the initial pixel values from Analyze images (so-called Window) was mapped to a range of 0 to 255 gray levels in the resulting BMP images. The Window parameter was chosen so as to cover only the full range of pixel values observed in the whole set of 


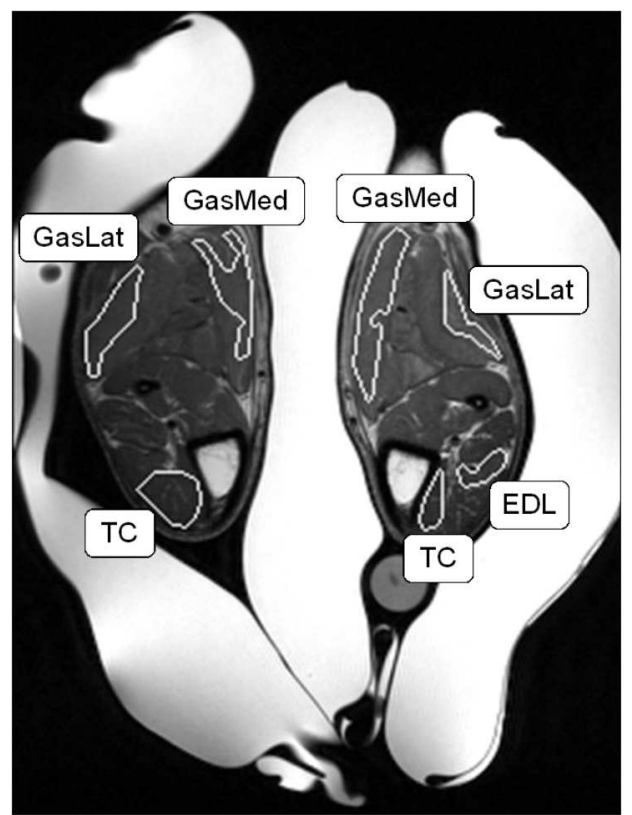

(a) GRMD dog

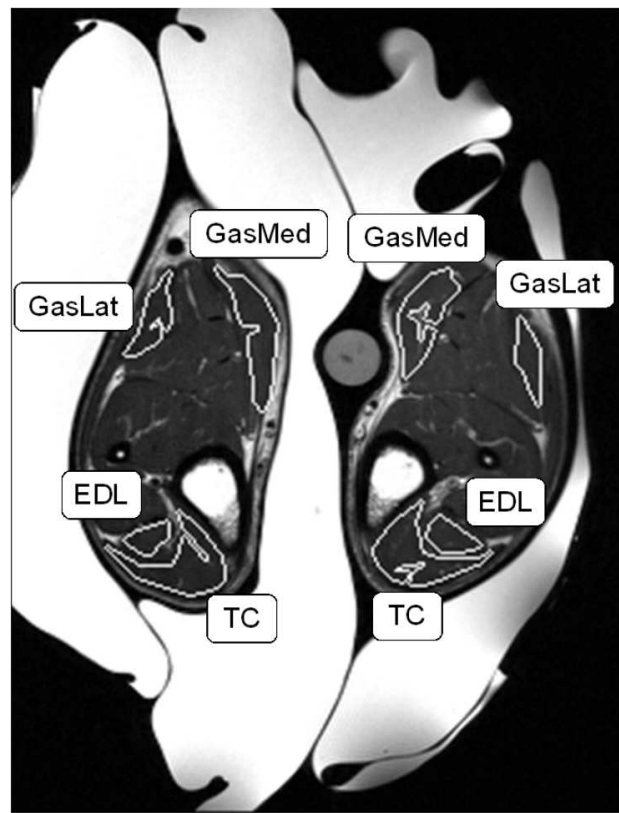

(b) healthy dog

Figure 1. Examples of T2-weighted MRI images, used in the experiments. The images were acquired on a GRMD $\operatorname{dog}$ (a) and one healthy control (b), at the age of 8 months. Delineated ROIs cover the four types of muscles considered in the study: EDL, GasLat, GasMed, and TC. Each of the ROIs has a size larger than 100 pixels

Table 2. Numbers of ROIs used in the experiments

\begin{tabular}{|c|c|c|c|c|c|}
\hline \multirow{2}{*}{ phase } & \multirow{2}{*}{ cohort type } & \multicolumn{4}{|c|}{ Muscle } \\
\cline { 3 - 6 } & & EDL & GasLat & GasMed & TC \\
\hline \multirow{2}{*}{ first } & healthy & 52 & 30 & 60 & 73 \\
& GRMD & 45 & 43 & 64 & 53 \\
\hline \multirow{2}{*}{ second } & healthy & 48 & 24 & 37 & 64 \\
& GRMD & 56 & 34 & 43 & 87 \\
\hline \multirow{2}{*}{ third } & healthy & 136 & 85 & 113 & 157 \\
& GRMD & 73 & 31 & 60 & 81 \\
\hline
\end{tabular}

the available ROIs. The original pixel values below the lower and above the upper limit of Window were transformed to 0 and 255, respectively. This allowed to obtain the maximum diversity of pixel gray levels in the analyzed ROIs. 
Table 3. Average sizes of ROIs used in the experiments

\begin{tabular}{|c|c|c|c|c|c|}
\hline \multirow{2}{*}{ phase } & \multirow{2}{*}{ cohort type } & \multicolumn{4}{|c|}{ Muscle } \\
\cline { 3 - 6 } & & EDL & GasLat & GasMed & TC \\
\hline \multirow{2}{*}{ first } & healthy & 202 & 161 & 290 & 205 \\
& GRMD & 156 & 189 & 293 & 165 \\
\hline \multirow{2}{*}{ second } & healthy & 239 & 184 & 395 & 255 \\
& GRMD & 189 & 220 & 379 & 250 \\
\hline \multirow{2}{*}{ third } & healthy & 279 & 220 & 426 & 316 \\
& GRMD & 160 & 199 & 328 & 236 \\
\hline
\end{tabular}

Then, eight different texture analysis methods (statistical, filter-based, and model-based) were applied in order to characterize the muscular tissue within each ROI. They were implemented in the homemade application Medical Image Processing (Duda, 2009). In total, 37 textural features were calculated:

- Avg (average), Var (variance), Skew (skewness), and Kurt (kurtosis) - first order statistics (abbreviated FOS), obtained from a gray level histogram,

- AngSecMom (angular second moment or energy), Contrast (contrast or inertia), Corr (correlation), SumSqr (sum of squares or variance), InvDiffMom (inverse difference moment or local homogeneity), SumAvg (sum average), SumVar (sum variance), SumEntr (sum entropy), Entr (entropy), DiffVar (difference variance), and DiffEntr (difference entropy), from the co-occurrence matrices (COM) introduced by Haralick et al. (1973),

- ShortEmph (short run emphasis), LongEmph (long run emphasis), GlNonUni (gray level non-uniformity), RlNonUni (run length nonuniformity), Fraction (fraction of image in runs), LowGlrEmph (low gray level runs emphasis), HighGlrEmph (high gray level runs emphasis), and RlEntr (run length entropy), from the run length matrices (RLM), a set of features proposed by Galloway (1975), then extended by Chu et al. (1990) and Albregtsen et al. (2000),

- GldmAngSecMom (angular second moment), GldmEntr (entropy), and GldmMean (mean), form the gray level difference matrices (GLDM) (Weszka et al., 1976),

- EntrE3L3, EntrS3L3, EntrS3E3, EntrE3E3, EntrS3S3 - entropy of an image region filtered, respectively, with the following pairs of Laws' masks: E3L3 and L3E3, S3L3 and L3S3, S3E3 and E3S3, E3E3 and E3E3, and S3S3 and S3S3 (LTE) (Laws, 1980), 
- GradAvg (average), GradVar (variance), GradSkew (skewness), and GradKurt (kurtosis), from the gradient matrix (GM) (Lerski et al., 1993),

- FractalDim - fractal dimension based on the fractional Brownian motion model (FB) described by Chen et al. (1998),

- AutoCorr - normalized autocorrelation coefficient (AC) (Gonzalez \& Woods, 2002).

Four standard directions of pixel runs $\left(0^{\circ}, 45^{\circ}, 90^{\circ}\right.$, and $\left.135^{\circ}\right)$ were considered when applying the COM, RLM, GLDM, FB, and AC methods. Since many ROIs were rather narrow and of an irregular shape, only the smallest possible distances between pairs of pixels ( 1 and 2 ) were taken into account for the COM, GLDM, FB and AC methods. In fact, with the use of larger distances, a rather considerable number of ROIs would have to be excluded from the analysis. This would have been undesirable due to the limited size of the available data set. Moreover, for the COM, RLM and GLDM methods, the number of gray levels was reduced from 256 to 64 , due to the fact that such a reduction proved to be the most advantageous, leading to the best classification results (Duda et al., 2016).

The co-occurrence matrices, run length matrices, and gray level difference matrices were constructed separately for each possible direction of pixel runs and (if applicable) for each of the two considered distances between pairs of pixels. Features calculated at different directions and/or distances were averaged.

Statistical Analysis. The statistical analyses concerned only those features that were obtained from healthy dogs. They were performed for each feature separately, in order to determine whether the differences in the feature values observed between two compared phases of canine growth were statistically significant. The following pairs of phases were considered: (i) the first and the second phase and (ii) the second and the third phase.

The choice of appropriate statistical tests turned out to be a non-trivial task. An important property of the database was that it contained images acquired repeatedly on the same individuals, at different moments of their lives. Effectively, the values of a feature derived from several examinations of the same individual might be related one to another. However, the use of tests for dependent variables became problematic due to the fact that each individual was examined a different number of times and at different ages, within the same phase of its growth. Furthermore, the numbers of ROIs (from which the features were calculated) varied from one examination to 


\section{Dorota Duda}

another. Finally, ROIs designated for each examination corresponded to several different slice locations within a given muscle. In this case, it was not possible to create triples of feature values (or $n$-tuples, with $n>3$ ) in which values at a given position would correspond to the same conditions of measurement: the same moment of an individual's life and the same image slice. Therefore, only information about the phase attributed to each value of feature was used. As the aim of this preliminary study was to find the features whose values might generally differ between phases of growth, regardless of the individual taken into account, the best possible solution seemed to be performing such tests as for independent samples.

In all the cases, the standard significance level $(\alpha=0.05)$ was used. First, the Shapiro-Wilk test was used to determine whether each of the two compared samples of feature values followed the normal distribution. If both samples met this condition, the T-test was applied to check if the mean values of two samples were statistically different from each other, at a significance level of 0.05 . Otherwise (if at least one of the samples did not meet this condition), the Mann-Whitney U-test was used to check the above. All the statistical analyses were performed with Statistica software, version 13.1 (StatSoft, Inc., 2016).

\section{Assessing the Relative Importance of Feature, Selection of} Features, and Classification. The relative importance (usefulness) of each textural feature in identifying the phase of dystrophy in GRMD dogs was assessed using the Monte Carlo procedure, initially described by Dramiński et al. (2008). Its modified version, used in this study, was proposed by Duda et al. (2016) and consisted in multiple repetitions of a single selection procedure, run on a "truncated" data set. Such data set was created by a random choice of $2 / 3$ initial observations and no more than $20 \%$ of the initially used features ( 6 features were always chosen here). A single selection procedure was repeated 250,000 times, each time with a different subset of observations and features, and was based on the best-first strategy with the forward search algorithm. During this process, a supervised, wrapper method (Kohavi \& John, 1997) combined with C4.5 tree (Quinlan, 1993) and 10-fold cross-validation was applied for the evaluation of each candidate subset of features.

After completing the entire MC selection procedure, the "incidence frequency rate" (IFR, the ratio between the number of cases in which the feature was selected and the number of times it occurred in the subsets of randomly chosen features in truncated data sets) was calculated for each feature and was considered as a determinant of feature usefulness. The features 
were then ranked according to their IFR values (from the most to the least selected) and different numbers of the top ranked features were tested. The subset of features ensuring the best identification of the dystrophy phase was finally assumed as optimal.

All the selection procedures were performed with Weka software (Hall et al., 2009). Additional tools for generating truncated data sets in the modified MC procedure and creating feature incidence frequency rankings were implemented by the author in $\mathrm{C}++$ language.

The usefulness of each of the obtained subsets of textural features (composed of: all tested features, all age-independent features, and features selected from among the age-independent ones) was assessed on the basis of the classification accuracy that the subset could ensure. Classification experiments were also performed with Weka. At this step, the following classifiers were separately used:

- Ensemble of Classifiers with an Adaptive Boosting voting scheme AdaBoostM1 (AB) (Freund \& Schapire, 1997) using C4.5 classifier as the underlying algorithm,

- back-propagation Neural Network (NN) (Bishop, 1995) with a sigmoidal activating function and one hidden layer wherein the number of neurons was equal to the average value of the number of features and the number of classes,

- Support Vector Machines (SVM) (Vapnik, 2000) using the Sequential Minimal Optimization (SMO) algorithm (Platt, 1998) and a seconddegree polynomial kernel.

Classification accuracies were assessed using 10-fold cross-validation, repeated 10 times. Each time, 100 partial results were averaged.

\section{Results and Discussion}

All the experiments were repeated separately for each of the considered types of muscles: EDL, GasLat, GasMed, and TC.

\section{Differentiation Between Phases of Growth in Healthy Dogs.} The classification accuracies (with standard deviation) for the preliminary experiment are presented in Table 4, separately for each classifier, i.e. Adaptive Boosting, Neural Network, and Support Vector Machines. Moreover, Table 4 contains (for comparison purposes) the classification results obtained when phases of dystrophy development in GRMD dogs were differentiated with all the possible features. 


\section{Dorota Duda}

It could be observed that the percentage of correctly identified phases of canine growth in healthy dogs is quite high, exceeding $84 \%$ for both the differentiation problems, i.e. (i) and (ii). This suggests that there may exist features that evolve with the dog's growth and that their differences between the adjacent phases may possibly be so large as to enable the correct identification of the dog's age. When comparing the results provided by the same classifier, for the same differentiation problem, and for the same type of muscle, it could be noticed that differentiation between phases of canine growth in healthy dogs is often more pronounced than differentiation between dystrophy phases in GRMD dogs (differences reaching up to $9.2 \%$ for problem (i) and up to $28.9 \%$ for problem (ii)). One could therefore suppose that a good "recognition of dystrophy phase" in GRMD dogs could actually be related to changes in muscles occurring with the dog's growth and not necessarily with dystrophy development. Nevertheless, it is not known if these are the same features that ensure a good recognition of the considered phases in healthy and in GRMD dogs. This justifies the need for further analyses.

Table 4. Classification accuracies (and standard deviations) [\%] achieved with the use of all the 37 initial features, for two differentiation problems: (i) the first phase vs. the second phase and (ii) the second phase vs. the third phase. The experiment consisted in identifying the phase of canine growth in healthy dogs and the phase of dystrophy development in GRMD dogs. The results were obtained by application of Adaptive Boosting (AB), Neural Network (NN), and Support Vector Machines (SVM) classifiers

\begin{tabular}{|c|c|c|c|c|c|c|}
\hline \multirow{2}{*}{ problem } & \multirow{2}{*}{ classifier } & \multirow{2}{*}{ cohort type } & \multicolumn{4}{|c|}{ Muscle } \\
\hline & & & EDL & GasLat & GasMed & $\mathrm{TC}$ \\
\hline \multirow{6}{*}{$\begin{array}{c}\text { (i) } \\
\text { first phase } \\
\text { vs. } \\
\text { second phase }\end{array}$} & \multirow{2}{*}{$\mathrm{AB}$} & healthy & $71.0(6.7)$ & $71.0(9.1)$ & $70.3(7.4)$ & $75.7(5.3)$ \\
\hline & & GRMD & $76.2(7.4)$ & $68.8(8.1)$ & $76.6(5.3)$ & $75.1(5.0)$ \\
\hline & \multirow{2}{*}{$\mathrm{NN}$} & healthy & $80.5(6.0)$ & $67.5(7.8)$ & $73.0(6.6)$ & $76.4(5.8)$ \\
\hline & & GRMD & $72.5(6.6)$ & $67.8(7.5)$ & $78.8(5.6)$ & $75.0(5.3)$ \\
\hline & \multirow{2}{*}{ SVM } & healthy & $84.5(5.2)$ & $71.3(8.9)$ & $80.1(6.1)$ & $82.6(5.5)$ \\
\hline & & GRMD & $75.3(6.7)$ & $70.3(7.9)$ & $81.6(5.9)$ & $76.0(5.1)$ \\
\hline \multirow{6}{*}{$\begin{array}{c}\text { (ii) } \\
\text { second phase } \\
\text { vs. } \\
\text { third phase }\end{array}$} & \multirow{2}{*}{$\mathrm{AB}$} & healthy & $78.5(3.7)$ & $73.3(5.6)$ & $79.5(4.1)$ & $80.3(3.3)$ \\
\hline & & GRMD & $72.8(5.8)$ & $59.3(9.6)$ & $65.6(7.2)$ & $69.5(5.6)$ \\
\hline & \multirow{2}{*}{$\mathrm{NN}$} & healthy & $76.0(4.1)$ & $81.5(5.9)$ & $80.3(4.2)$ & $75.9(4.3)$ \\
\hline & & GRMD & $68.2(6.6)$ & $52.6(9.2)$ & $64.7(7.5)$ & $71.9(5.0)$ \\
\hline & \multirow{2}{*}{ SVM } & healthy & $78.6(4.0)$ & $76.0(5.2)$ & $84.2(3.8)$ & $80.1(3.5)$ \\
\hline & & GRMD & $79.9(5.3)$ & $60.1(9.3)$ & $62.0(7.6)$ & $74.5(4.8)$ \\
\hline
\end{tabular}


Evaluating the Usefulness of Each Feature and Feature Selection on Age-Independent Features. The results for the second and the third experiment are combined and presented in common tables: in Table 5 for the first (i) differentiation problem (the first phase vs. the second phase) and in Table 6 - for the second (ii) differentiation problem (the second phase vs. the third phase). For each table, the features with statistically significant differences $(\alpha=0.05)$ between values in two considered phases of growth in healthy dogs are marked with an em dash ("-"). These were eliminated from further analyses. The remaining features were used in differentiation between phases of dystrophy progression in affected dogs. The corresponding numbers are the frequencies of selections (IFR) in the modified Monte Carlo procedure.

It can be seen that sets of features considered to be dependent on the dog's age are different for each differentiation problem, (i) and (ii) and vary between the four analyzed types of muscles, i.e. EDL, GasLat, GasMed and TC. For example, when the first two phases of the dog's growth were differentiated based on EDL's texture - only 8 features (of the 37 tested) showed statistically significant differences between phases. However, when distinguishing between the second and third phase, based on the same muscle, as many as 20 features demonstrated significant differences. Only 3 features belonged to the common part of the aforementioned feature sets. In turn, TC muscle provided 24 and 7 textural features differing from one phase to another, respectively for the first (i) and the second (ii) differentiation problem. The obtained results suggest that some features can only evolve from the dog's birth to a certain moment of its life, while other features begin to change only after the dog reaches a certain age. Moreover, they indicate that the texture of each muscle type in a given phase of the dog's growth can have different properties. Therefore, it would not be advisable to process all muscles at a time, ignoring their type.

Finally, it can be noted that many textural features identified in the referenced works as "potentially useful" for the characterization of dystrophy development turned out to be age-dependent and thus were designated for elimination. In some cases, this concerned as many as over half the number of COM-, RLM-, and GLDM-based features (see, for example, the results for GasMed and TC muscles in the first differentiation problem (Table 5) or GasMed muscle in the second differentiation problem (Table 6)). As for the remaining (age-independent) COM-, or RLM-based features, they still demonstrated their usefulness, being quite frequently selected by the modified MC procedure. Depending on the differentiation problem, other features were also present in the selected subsets of features, e.g. FOS - for problem (i) or LTE-based - for problem (ii). 
Table 5. The first (i) differentiation problem: the first phase vs. the second phase. Features marked by an em dash ("_") demonstrated statistically significant differences between the considered phases of growth in healthy dogs $(\alpha=0.05)$. The remaining features were used in the identification of dystrophy phases in GRMD dogs. The corresponding numbers are incidences of selections [\%] in the modified MC procedure

\begin{tabular}{|c|c|c|c|c|}
\hline \multirow{2}{*}{ Feature } & \multicolumn{4}{|c|}{ Muscle } \\
\hline & EDL & GasLat & GasMed & $\mathrm{TC}$ \\
\hline$A v g$ & - & - & 21.16 & - \\
\hline Var & 29.96 & 35.47 & - & - \\
\hline Skew & 28.34 & - & 38.23 & 27.06 \\
\hline Kurt & 60.79 & 12.97 & 34.83 & - \\
\hline AngSecMom & 68.53 & 22.40 & - & 34.37 \\
\hline Contrast & 46.47 & 63.59 & - & - \\
\hline Corr & 10.04 & 12.17 & 25.08 & 15.37 \\
\hline SumSqr & 19.42 & 56.07 & - & - \\
\hline InvDiffMom & 81.42 & 29.58 & - & - \\
\hline SumAvg & - & - & 28.67 & - \\
\hline SumVar & 17.04 & 17.19 & - & 22.82 \\
\hline SumEntr & 27.26 & 22.84 & - & 14.74 \\
\hline Entr & 33.76 & 18.45 & - & 74.78 \\
\hline DiffVar & 39.60 & 63.41 & 62.41 & - \\
\hline DiffEntr & 32.87 & 56.41 & - & - \\
\hline ShortEmph & 15.66 & 26.51 & 一 & 一 \\
\hline LongEmph & 48.76 & 23.79 & - & - \\
\hline GlNonUni & 42.58 & 19.41 & - & - \\
\hline RlNonUni & 15.88 & 21.12 & - & 61.92 \\
\hline Fraction & 30.68 & 19.02 & - & - \\
\hline LowGlrEmph & - & - & 27.44 & - \\
\hline HighGlrEmph & - & - & 17.87 & - \\
\hline RlEntr & - & 25.74 & 72.54 & 27.47 \\
\hline GldmAngSecMom & 57.24 & 24.89 & - & - \\
\hline GldmEntr & 32.30 & 46.27 & - & - \\
\hline GldmMean & 36.35 & 32.37 & - & - \\
\hline EntrE3L3 & 31.89 & 11.45 & - & 20.89 \\
\hline EntrS3L3 & 19.31 & - & 一 & - \\
\hline EntrS3E3 & 19.71 & 16.06 & - & 15.67 \\
\hline EntrE3E3 & 45.51 & 19.51 & - & 17.86 \\
\hline EntrS3S3 & - & 21.00 & - & 15.36 \\
\hline GradAvg & 50.99 & 43.30 & - & - \\
\hline GradVar & - & 26.60 & 60.13 & - \\
\hline GradSkew & 60.22 & 10.77 & 25.76 & - \\
\hline GradKurt & 48.04 & 8.64 & 18.15 & - \\
\hline FractalDim & 9.84 & 9.03 & 24.37 & - \\
\hline AutoCorr & - & 21.59 & - & 11.83 \\
\hline
\end{tabular}


Table 6. The second (ii) differentiation problem: the second phase vs. the third phase. Features marked by an em dash ("—") demonstrated statistically significant differences between the considered phases of growth in healthy dogs $(\alpha=0.05)$. The remaining features were used in the identification of dystrophy phases in GRMD dogs. The corresponding numbers are incidences of selections [\%] in the modified MC procedure

\begin{tabular}{|c|c|c|c|c|}
\hline \multirow{2}{*}{ Feature } & \multicolumn{4}{|c|}{ Muscle } \\
\hline & EDL & GasLat & GasMed & $\mathrm{TC}$ \\
\hline$A v g$ & 50.51 & - & - & 53.61 \\
\hline Var & - & 15.71 & 19.47 & 38.70 \\
\hline Skew & - & 24.08 & 14.85 & - \\
\hline Kurt & - & 13.49 & 12.53 & - \\
\hline AngSecMom & 12.26 & - & - & 16.37 \\
\hline Contrast & - & 12.34 & 一 & 15.46 \\
\hline Corr & 17.16 & 13.44 & - & 19.30 \\
\hline SumSqr & - & 33.09 & 17.58 & 27.14 \\
\hline InvDiffMom & 9.70 & 一 & - & 42.20 \\
\hline SumAvg & 55.86 & - & - & 56.22 \\
\hline SumVar & - & 9.50 & 12.83 & 22.19 \\
\hline SumEntr & 10.17 & 10.55 & 17.70 & 29.57 \\
\hline Entr & 23.12 & 15.34 & 35.52 & 17.84 \\
\hline DiffVar & - & 10.54 & 20.90 & 22.10 \\
\hline DiffEntr & - & 24.89 & - & 27.52 \\
\hline ShortEmph & 9.56 & - & - & 11.79 \\
\hline LongEmph & 16.99 & - & - & 11.79 \\
\hline GlNonUni & 34.10 & 一 & 39.27 & - \\
\hline RlNonUni & - & 36.78 & 71.41 & - \\
\hline Fraction & 14.75 & - & - & 13.94 \\
\hline LowGlrEmph & 47.15 & - & - & 67.00 \\
\hline HighGlrEmph & 56.93 & - & - & 42.59 \\
\hline RlEntr & - & 23.09 & 11.27 & 9.37 \\
\hline GldmAngSecMom & 24.16 & 17.55 & - & 29.87 \\
\hline GldmEntr & - & 26.73 & - & 23.90 \\
\hline GldmMean & - & 18.83 & - & 28.64 \\
\hline EntrE3L3 & - & 27.91 & 20.75 & 13.83 \\
\hline EntrS3L3 & 15.78 & 20.75 & - & 23.69 \\
\hline EntrS3E3 & 23.20 & 26.52 & - & 9.49 \\
\hline EntrE3E3 & - & 14.02 & 82.40 & 10.45 \\
\hline EntrS3S3 & 32.42 & 43.41 & - & 9.41 \\
\hline GradAvg & - & 13.50 & 13.59 & 22.69 \\
\hline GradVar & - & 9.40 & 15.43 & - \\
\hline GradSkew & - & - & - & - \\
\hline GradKurt & - & - & - & - \\
\hline FractalDim & - & 7.07 & - & 18.57 \\
\hline AutoCorr & - & 21.62 & 15.14 & 16.41 \\
\hline
\end{tabular}




\section{Classification-Based Evaluation of Different Sets of Age-} Independent Features. At the end, two sets of age-independent features were used for the characterization of dystrophic tissue in the differentiation between the consecutive phases of dystrophy development: (i) the first phase vs. the second phase and (ii) the second phase vs. the third phase. The first set comprised of all the features independent of the dog's age, whereas the second one constituted the best combination of the top-ranked features from the feature incidence frequency ranking created by the MC procedure. Classification accuracies (with standard deviation) obtained with the use of these sets are presented in Table 7. As in the preliminary experiment, differentiation was performed with the use of three classifiers, i.e. Adaptive Boosting, Neural Network, and Support Vector Machines.

Table 7. Classification accuracies (and standard deviations) [\%] achieved with age-independent features, separately for each differentiation problem: (i) the first phase vs. the second phase and (ii) the second phase vs. the third phase. The tested sets were composed of: all the features independent of the dog's age (denoted "all") and the features selected applying the modified MC procedure (optimal set, denoted "max MC"). For the latter case, the number of the top-ranked features for which the best classification result was achieved is given in square brackets. The results were obtained by application of the AB, NN, and SVM classifiers

\begin{tabular}{|c|c|c|c|c|c|c|}
\hline \multirow{2}{*}{ problem } & \multirow{2}{*}{ classifier } & \multirow{2}{*}{ feature set } & \multicolumn{4}{|c|}{ Muscle } \\
\hline & & & EDL & GasLat & GasMed & TC \\
\hline \multirow{3}{*}{$\begin{array}{c}\text { (i) } \\
\text { first } \\
\text { phase } \\
\text { vs. } \\
\text { second } \\
\text { phase }\end{array}$} & $\mathrm{AB}$ & $\begin{array}{c}\text { all } \\
\max \mathrm{MC}\end{array}$ & $\begin{array}{c}76.5(6.5) \\
77.8(6.7) \\
{[24]}\end{array}$ & $\begin{array}{c}66.8(8.0) \\
72.9(7.1) \\
{[6]}\end{array}$ & $\begin{array}{c}71.3(6.7) \\
71.9(6.7) \\
{[6]}\end{array}$ & $\begin{array}{c}69.4(5.8) \\
70.8(5.3) \\
{[4]}\end{array}$ \\
\hline & NN & $\begin{array}{c}\text { all } \\
\max \mathrm{MC}\end{array}$ & $\begin{array}{c}72.4(6.5) \\
79.4(6.5) \\
{[12]}\end{array}$ & $\begin{array}{c}66.2(8.2) \\
74.9(6.4) \\
{[1]}\end{array}$ & $\begin{array}{c}74.4(5.8) \\
78.1(5.7) \\
{[7]}\end{array}$ & $\begin{array}{c}69.3(6.0) \\
75.9(5.5) \\
{[3]}\end{array}$ \\
\hline & SVM & $\begin{array}{c}\text { all } \\
\max \mathrm{MC}\end{array}$ & $\begin{array}{c}76.9(6.7) \\
80.0(7.1) \\
{[14]}\end{array}$ & $\begin{array}{c}73.7(9.2) \\
78.1(8.4) \\
{[25]}\end{array}$ & $\begin{array}{c}77.3(5.5) \\
77.3(5.5) \\
{[13]}\end{array}$ & $\begin{array}{c}72.6(5.3) \\
74.9(5.3) \\
{[10]}\end{array}$ \\
\hline \multirow{3}{*}{$\begin{array}{c}\text { (ii) } \\
\text { second } \\
\text { phase } \\
\text { vs. } \\
\text { third } \\
\text { phase }\end{array}$} & $\mathrm{AB}$ & $\begin{array}{c}\text { all } \\
\max \mathrm{MC}\end{array}$ & $\begin{array}{c}68.8(5.9) \\
71.1(6.1) \\
{[7]}\end{array}$ & $\begin{array}{c}57.8(8.2) \\
59.6(8.5) \\
{[12]}\end{array}$ & $\begin{array}{c}67.5(6.3) \\
72.2(5.7) \\
{[3]}\end{array}$ & $\begin{array}{c}68.4(5.3) \\
71.6(5.9) \\
{[23]}\end{array}$ \\
\hline & $\mathrm{NN}$ & $\begin{array}{c}\text { all } \\
\max \mathrm{MC}\end{array}$ & $\begin{array}{c}68.4(6.3) \\
75.2(5.4) \\
{[6]} \\
\end{array}$ & $\begin{array}{c}61.2(9.1) \\
66.2(8.2) \\
{[6]} \\
\end{array}$ & $\begin{array}{c}68.6(7.3) \\
71.9(6.5) \\
{[3]} \\
\end{array}$ & $\begin{array}{c}73.3(5.9) \\
77.6(5.2) \\
{[8]}\end{array}$ \\
\hline & SVM & $\begin{array}{c}\text { all } \\
\max \mathrm{MC}\end{array}$ & $\begin{array}{c}75.1(5.4) \\
78.5(5.6) \\
{[11]}\end{array}$ & $\begin{array}{c}61.6(9.0) \\
64.8(8.9) \\
{[14]}\end{array}$ & $\begin{array}{c}70.9(6.8) \\
72.5(5.9) \\
{[8]}\end{array}$ & $\begin{array}{c}73.5(5.0) \\
76.0(4.6) \\
{[22]}\end{array}$ \\
\hline
\end{tabular}


First, the results obtained using the whole set of features independent of the dog's age were compared to those obtained using all the 37 features considered in the study (the latter ones could be found in Table 4). It could be observed, that the classification accuracies obtained with these two sets do not differ too much. For the first (i) differentiation problem (the first phase vs. the second phase), eliminating features that evolve along with the dog's growth led more often to a slight worsening of the classification accuracies (up to 5.7\%), in comparison to the results obtained for the whole set of 37 features. However, in most cases this worsening was not statistically significant at a significance level of 0.05 . For the second (ii) differentiation problem (the second phase vs. the third phase), eliminating the age-dependent features very often led to an improvement of classification accuracy, form $0.2 \%$ (not statistically significant) to $8.9 \%$ (statistically significant at a significance level of 0.05) and the maximum worsening of classification results was $4.8 \%$.

The application of feature selection based on the modified Monte Carlo procedure always resulted in the amelioration of classification results, up to $8.7 \%$, in comparison to results corresponding to the use of all the age-independent features (apart from only one exception - no difference observed). Even if sometimes this amelioration was not statistically significant, a significant reduction in the size of feature sets was at least achieved. It is also with the MC strategy, that the best overall classification result with age-independent features was observed, both for the first and for the second differentiation problem - respectively $80.0 \%$ and $78.5 \%$ of correctly identified phases of dystrophy progression (always with the SVM classifier and for EDL muscle). Such results are inferior to these obtained with all the 37 features tested (dependent on and independent of the dog's age) by, respectively, $1.6 \%$ and $1.4 \%$, but these differences are not statistically significant.

\section{Conclusions and Future Work}

The study assessed the possibility of applying MRI texture analysis for evaluating Duchenne Muscular Dystrophy therapies. The work was based on the popular Golden Retriever Muscular Dystrophy canine model, in which three phases of canine growth and/or dystrophy development were considered: the first phase ( $0-4$ months of age), the second phase (from over 4 to 6 months), and the third phase (from over 6 months to death). Two differentiation problems were posed, i.e. (i) the first phase vs. the second phase 


\section{Dorota Duda}

and (ii) the second phase vs. the third phase. The main objective of the work was to investigate which of the commonly used textural features can evolve as the dog grows. It was assumed that the use of such features could alter the right assessment of dystrophy progression or its response to treatment. In total, 37 features, derived from T2-weighted images were tested. 8 different TA methods (statistical, filter-based, and model-based) were considered. Experiments were conducted separately for four types of muscles: EDL, GasLat, GasMed, and TC. Statistical tests (the Mann-Whitney U-test and the T-test) were used for finding the age-dependent features. The modified Monte Carlo procedure was used on the remaining features in order to find the best set of features independent of the dog's age. Three classifiers (Adaptive Boosting, Neural Network, Support Vector Machines) were finally applied for the evaluation of different sets of tested features.

The experiments showed that there exists a relatively large group of textural features whose values change with the growth of the dog. Many of them only evolve over a certain period of the dog's life (at the transition from the first phase to the second phase, or from the second phase to the third phase). Such features were eliminated from further investigation (a separate set for each differentiation problem). It was found that with proper selection of features (from among the features independent of the dog's age) the classification qualities in the identification of dystrophy phases in GRMD dogs do not differ significantly from the results obtained for all the 37 features tested. The subsets of features obtained with the modified MC procedure ensured $80.0 \%$ and $78.5 \%$ of correctly identified phases of dystrophy progression, respectively for the first (i) and second (ii) differentiation problem. These results were obtained using the SVM classifier, for the EDL muscle.

The approach adapted in the present study, consisting in the elimination of features evolving with the dog's growth, could be a satisfactory solution only for the problem of differentiating between the adjacent phases of dystrophy. However, it might not be a good enough concept in a situation when more than two phases of dystrophy progression are to be identified. In fact, the sets of features whose values differ significantly among the three phases of growth in healthy dogs were relatively small, regardless of muscle type. A reasonable idea would be to build a model describing the differences between feature evolution with the dog's growth and dystrophy progression. However, testing such a model could turn out to be difficult if it is not known how slowing down the disease may affect the summary evolution of each feature.

In the future, if texture analysis were to be applied to test therapeutic effects, experiments should undoubtedly be done on a much larger data set. 
The use of other MRI series (such as T1-weighted ones) should also be considered. It would be desirable to include more textural features in analyses, especially model- or filter-based. Taking into account the fact that features evolve in different ways for each type of muscle, a reasonable idea would be to treat different types of muscles simultaneously. Finally, other methods can be applied for assessing the usefulness of features in testing the effects of a therapy (indicating the features that may evolve with the dog's growth), e.g. based on the feature incidence frequency rate in the MC procedure while the dog's age is identified.

\section{Acknowledgements}

I would like to thank Noura Azzabou from the Institute of Myology, Nuclear Magnetic Resonance Laboratory, Paris, France for preparing and providing the database of images and ROIs on which the experiments were performed in this study. I also thank Jacques D. de Certaines and other participants of the European COST Action BM1304, MYO-MRI for valuable comments and discussions.

This work was supported by grant $\mathrm{S} / \mathrm{WI} / 2 / 18$ (from the Bialystok University of Technology, Bialystok, Poland), founded by the Polish Ministry of Science and Higher Education.

\section{R E F E R E N C E S}

Albregtsen, F., Nielsen, B., \& Danielsen, H. E. (2000). Adaptive gray level run length features from class distance matrices. In A. Sanfeliu, J. J. Villanueva, M. Vanrell, R. Alqukzar, J. Crowley, \& Y. Shirai (Eds.), Proceedings 15th International Conference on Pattern Recognition. ICPR-2000. Vol. 3. Image, Speech, and Signal Processing (pp. 738-741). doi: 10.1109/ICPR.2000.903650

Birnkrant, D. J., Bushby, K., Bann, C. M., Alman, B. A., Apkon, S. D., Blackwell, A., Case, L. E., et al. (2018a). Diagnosis and management of Duchenne muscular dystrophy, part 2: respiratory, cardiac, bone health, and orthopaedic management. Lancet Neurology, 17(4), 347-361. doi: 10.1016/S14 74-4422(18)30025-5

Birnkrant, D. J., Bushby, K., Bann, C. M., Apkon, S. D., Blackwell, A., Brumbaugh, D., Case, L. E., et al. (2018b). Diagnosis and management of Duchenne muscular dystrophy, part 1: diagnosis, and neuromuscular, rehabilitation, endocrine, and gastrointestinal and nutritional management. Lancet Neurology, 17(3), 251-267. doi: 10.1016/S1474-4422(18)30024-3

Bishop, C. M. (1995). Neural networks for pattern recognition. Oxford, United Kingdom: Clarendon Press. 


\section{Dorota Duda}

Chen, E. L., Chung, P.-C., Chen, C.-L., Tsai, H.-M., \& Chang, C.-I. (1998). An automatic diagnostic system for CT liver image classification. IEEE Transactions on Biomedical Engineering, 45(6), 783-794. doi: 10.1109/10.678613

Chu, A., Sehgal, C. M., \& Greenleaf, J. F. (1990). Use of gray value distribution of run lengths for texture analysis. Pattern Recognition Letters, 11(6), 415-419. doi: 10.1016/0167-8655(90)90112-F

de Certaines, J. D., Larcher, T., Duda, D., Azzabou, N., Eliat, P.-A., Escudero, L. M., Pinheiro, A. M. G., et al. (2015). Application of texture analysis to muscle MRI: 1-What kind of information should be expected from texture analysis? EPJ Nonlinear Biomedical Physics, 3:3. doi: 10.1140/epjnbp/ s40366-015-0017-1

Dramiński, M., Rada-Iglesias, A., Enroth, S., Wadelius, C., Koronacki, J., \& Komorowski, J. (2008). Monte Carlo feature selection for supervised classification. Bioinformatics, 24(1), 110-117. doi: 10.1093/bioinformatics/btm486

Duda, D. (2009). Classification d'images médicales basée sur l'analyse de texture (Unpublished doctoral dissertation). University of Rennes 1, Rennes, France.

Duda, D., Kretowski, M., Azzabou, N., \& de Certaines, J. D. (2015). MRI texture analysis for differentiation between healthy and Golden Retriever Muscular Dystrophy dogs at different phases of disease evolution. In K. Saeed \& W. Homenda (Eds.), Computer Information Systems and Industrial Management. CISIM 2015 (pp. 255-266). Lecture Notes in Computer Science: Vol. 9339. Springer, Cham. doi: 10.1007/978-3-319-24369-6_21

Duda, D., Kretowski, M., Azzabou, N., \& de Certaines, J. D. (2016). MRI texturebased classification of dystrophic muscles. A search for the most discriminative tissue descriptors. In K. Saeed \& W. Homenda (Eds.), Computer Information Systems and Industrial Management. CISIM 2016 (pp. 116128). Lecture Notes in Computer Science: Vol. 9842. Springer, Cham. doi: 10.1007/978-3-319-45378-1_11

EMA (2015). Guideline on the clinical investigation of medicinal products for the treatment of Duchenne and Becker muscular dystrophy. European Medicines Agency, Committee for Medicinal Products for Human Use. Retrieved from http://www.ema.europa.eu/docs/en_GB/document_library/Scientific_guide line/2015/12/WC500199239.pdf.

Fan, Z., Wang, J., Ahn, M., Shiloh-Malawsky, Y., Chahin, N., Elmore, S., Bagnell, C. R., et al. (2014). Characteristics of magnetic resonance imaging biomarkers in a natural history study of golden retriever muscular dystrophy. Neuromuscular Disorders, 24(2), 178-191. doi: 10.1016/j.nmd.2013.10.005

Finanger, E. L., Russman, B., Forbes, S. C., Rooney, W. D., Walter, G. A., \& Vandenborne, K. (2012). Use of skeletal muscle MRI in diagnosis and monitoring disease progression in Duchenne Muscular Dystrophy. Physical Medicine and Rehabilitation Clinics of North America, 23(1), 1-10. doi: 10.1016/j.pmr.2011.11.004 
Freund, Y., \& Schapire, R. E. (1997). A decision-theoretic generalization of on-line learning and an application to boosting. Journal of Computer and System Science, 55(1), 119-139. doi: 10.1006/jcss.1997.1504

Galloway, M. M. (1975). Texture analysis using gray level run lengths. Computer Graphics and Image Processing, 4(2), 172-179. doi: 10.1016/S0146$664 \mathrm{X}(75) 80008-6$

Gonzalez, R. C., \& Woods, R. E. (2002). Digital Image Processing (2nd ed.). Boston, MA, USA: Addison-Wesley Longman Publishing Co., Inc.

Guiraud, S., Aartsma-Rus, A., Vieira, N. M., Davies, K. E., van Ommen, G.J. B., \& Kunkel, L. M. (2015). The pathogenesis and therapy of muscular dystrophies. Annual Review of Genomics and Human Genetics, 16, 281-308. doi: 10.1146/annurev-genom-090314-025003

Hall, M., Frank, E., Holmes, G., Pfahringer, B., Reutemann, P., \& Witten, I. H. (2009). The WEKA data mining software: an update. ACM SIGKDD Explorations Newsletter, 11(1), 10-18. doi: 10.1145/1656274.1656278

Haralick, R. M., Shanmugam, K., \& Dinstein, I. (1973). Textural Features for Image Classification. IEEE Transactions on Systems, Man, and Cybernetics, SMC3(6), 610-621. doi: 10.1109/TSMC.1973.4309314

Kohavi, R., \& John, G. H. (1997). Wrappers for Feature Subset Selection. Artificial Intelligence, 97(1-2), 273-324. doi: 10.1016/S0004-3702(97)00043-X

Kornegay, J. N. (2017). The golden retriever model of Duchenne muscular dystrophy. Skeletal Muscle, \%:9. doi: 10.1186/s13395-017-0124-z

Laws, K. I. (1980). Textured image segmentation (Unpublished doctoral dissertation). University of Southern California, USA.

Lerski, R. A., de Certaines, J. D., Duda, D., Klonowski, W., Yang, G., Coatrieux, J. L., Azzabou, N., \& Eliat, P. A. (2015). Application of texture analysis to muscle MRI: 2 - technical recommendations. EPJ Nonlinear Biomedical Physics, 3:2. doi: 10.1140/epjnbp/s40366-015-0018-0

Lerski, R. A., Straughan, K., Shad, L., Boyce, D., Bluml, S., \& Zuna, I. (1993). MR image texture analysis - an approach to tissue characterization. Magnetic Resonance Imaging, 11(6), 873-887. doi: 10.1016/0730-725X(93)902 05-R

LoMauro, A., d'Angelo, M. G., \& Aliverti, A. (2015). Assessment and management of respiratory function in patients with Duchenne muscular dystrophy: current and emerging options. Therapeutic and Clinical Risk Management, 11, 1475-1488. doi: 10.2147/TCRM.S55889

Martins-Bach, A. B., Malheiros, J., Matot, B., Martins, P. C. M., Almeida, C. F., Caldeira, W., Ribeiro, A. F., et al. (2015). Quantitative T2 combined with texture analysis of nuclear Magnetic Resonance Images identify different degrees of muscle involvement in three mouse models of muscle dystrophy: mdx, Large(myd) and mdx/Large(myd). PLOS ONE, 10(2): e0117835. doi: 10.1371/journal.pone.0117835 


\section{Dorota Duda}

National Research Council (2011). Guide for the Care and Use of Laboratory Animals. Washington, DC: The National Academies Press. doi: 10.17226/12910

Platt, J. C. (1998). Fast Training of Support Vector Machines using Sequential Minimal Optimization. In B. Scholkopf, C. J. C. Burges, \& A. J. Smola (Eds.), Advances in Kernel Methods - Support Vector Learning (pp. 185208). Cambridge, MA, USA: MIT Press.

Quinlan, J. R. (1993). C4.5: Programs for Machine Learning. San Francisco, CA, USA: Morgan Kaufmann Publishers Inc.

Salmaninejad, A., Valilou, S. F., Bayat, H., Ebadi, N., Daraei, A., Yousefi, M., Nesaei, A., \& Mojarrad, M. (2018). Duchenne muscular dystrophy: an updated review of common available therapies. International Journal of Neuroscience, 128(9), 854-864. doi: 10.1080/00207454.2018.1430694

Shieh, P. B. (2015). Duchenne muscular dystrophy: clinical trials and emerging tribulations. Current Opinion in Neurology, 28(5), 542-546. doi: 10.1097/ WCO.0000000000000243

Simon, N. G., Noto, Y.-I., \& Zaidman, C. M. (2016). Skeletal muscle imaging in neuromuscular disease. Journal of Clinical Neuroscience, 33, 1-10. doi: 10.1016/j.jocn.2016.01.041

Spurney, C. F. (2011). Cardiomyopathy of Duchenne muscular dystrophy: current understanding and future directions. Muscle \& Nerve, 44(1), 8-19. doi: 10.1002/mus.22097

Thibaud, J. L., Azzabou, N., Barthelemy, I., Fleury, S., Cabrol, L., Blot, S., \& Carlier, P. G. (2012). Comprehensive longitudinal characterization of canine muscular dystrophy by serial NMR imaging of GRMD dogs. Neuromuscular Disorders, 22 (Suppl. 2), S85-S99. doi: 10.1016/j.nmd.2012.05.010

Vapnik, V. N. (2000). The nature of statistical learning theory (2nd ed.). New York, USA: Springer. doi: 10.1007/978-1-4757-3264-1

Wang, J., Fan, Z., Vandenborne, K., Walter, G., Shiloh-Malawsky, Y., An, H., Kornegay, J. N., \& Styner, M. A. (2013). A computerized MRI biomarker quantification scheme for a canine model of Duchenne muscular dystrophy. International Journal. of Computer Assisted Radiology and Surgery, 8(5), 763-774. doi: $10.1007 / \mathrm{s} 11548-012-0810-6$

Weszka, J. S., Dyer, C. R., \& Rosenfeld, A. (1976). A comparative study of texture measures for terrain classification. IEEE Transactions on Systems, Man, and Cybernetics, SMC-6(4), 269-285. doi: 10.1109/TSMC.1976.5408777

Yang, G., Lalande, V., Chen, L., Azzabou, N., Larcher, T., de Certaines, J. D., Shu, H., \& Coatrieux, J. L. (2015). MRI texture analysis of GRMD dogs using orthogonal moments: A preliminary study. IRBM, 36(4), 213-219. doi: 10.1016/j.irbm.2015.06.004 